\title{
Mecanismos de acción de agentes propuestos para el tratamiento farmacológico específico de la infección por SARS-CoV-2
}

\author{
BASTIÁN ABARCA R.*, PAVAN DADLANI M.**, JORGE WIDERSTRÖM I.**, \\ JOCELYN VARGAS U.*** y JAVIER GARCÍA G.****
}

\author{
Mechanisms of action of proposed agents for the specific pharmacological treatment \\ of SARS-CoV-2 infection
}

Since the beginning of the pandemic by SARS-CoV-2, the pathogen responsible for COVID-19, many of the therapeutic options for its management have been under constant revision, in order to verify their safety and efficiency. Knowledge of the viral structure and pathogenesis make it possible to determine the molecular pathways that may be targeted with current available drugs. The experience with these drugs comes mainly from infections caused by SARS-CoV and MERS-CoV, in vitro studies with SARS-CoV-2 that yield variable results, and clinical experience that does not ensure effectiveness and safety of such drugs. To date, it has not been possible to elucidate a specific treatment scheme, because of the constant release of evidence that challenges the usefulness of the proposed drugs. This has motived us to continue seeking for an effective strategy that allows to manage this pandemic in a safe and efficient manner, so that therapeutic benefit surpasses the related adverse drug reactions that can occur. The following review aims to showcase the evidence available to date by defining the activity of each drug based on its mechanism of action.

Key words: SARS-CoV-2; CoVID-19; Coronavirus Disease; Causality; Viral Structures.

\section{Resumen}

Desde la notificación de la pandemia por SARS-CoV-2, agente patógeno responsable del COVID-19, muchos de los tratamientos dirigidos a su manejo han estado sometidos a estudios de manera constante, con el fin de comprobar su eficacia y seguridad. El conocimiento de su virología y etiopatogenia posibilitaría objetivar los pasos moleculares especificos que puedan ser blancos terapéuticos de variados fármacos actualmente disponibles. Esta experiencia proviene principalmente de las infecciones por SARS-CoVy MERS-CoV, con resultados variados 'in vitro' en el SARS-CoV-2, sin evidencia clínica que demuestre efectividad y seguridad de dichos tratamientos. A la fecha, no se ha podido concretar con claridad un esquema de tratamiento específico, debido a que la evidencia surgida ha puesto en jaque cada uno de los fármacos propuestos. Esto ha motivado a continuar en la búsqueda de una estrategia efectiva que permita manejar esta pandemia con la seguridad y eficacia necesaria para que el beneficio terapéutico esté por sobre los posibles efectos adversos que estos esquemas farmacológicos pudiesen presentar. La siguiente revisión pretende mostrar la evidencia disponible a la fecha, definiendo la actividad de cada fármaco en función de su mecanismo de acción.

Palabras clave: SARS-CoV-2; CoVID-19; enfermedad por coronavirus; causalidad; estructura viral.

\footnotetext{
* Médico-Cirujano, Facultad de Medicina, Universidad San Sebastián. Santiago, Chile.

** Interno de Medicina, Facultad de Medicina, Universidad San Sebastián. Santiago, Chile.

*** Enfermera, Unidad Coronaria, Depto. Cardiovascular, Hospital Clínico Universidad de Chile. Santiago, Chile.

**** Médico de Urgencia. Unidad Coronaria de Intermedio. Depto. Cardiovascular, Hospital Clínico Universidad de Chile. Santiago, Chile.
} 


\section{Introducción}

Este es un tema en constante evolución, por lo que todo concepto vertido a continuación puede variar de forma dinámica a disposición de la evidencia científica disponible. Desde el anuncio de la nueva pandemia el 11 marzo de $2020^{1}$ a causa del coronavirus 2 del síndrome respiratorio agudo severo (SARS-CoV-2), agente responsable de causar la enfermedad del coronavirus 2019 (COVID-19) ${ }^{2}$, ha existido controversia al definir una terapia efectiva que detenga su progresión. Conocer su patogenia sin duda es un avance tecnológico importante, pues sienta las bases para definir los blancos moleculares de un posible tratamiento.

En esta revisión se buscará plasmar la evidencia disponible a la fecha, definiendo la actividad de cada fármaco en función de su mecanismo de acción. Para ello, se revisará en detalle la patogenia del SARS-CoV-2, en un intento de introducir los probables blancos moleculares. No se incluyeron detalles sobre el ajuste de dosis de cada esquema, así como tampoco se discutirá la farmacovigilancia ni detalles de la preparación o administración de estos medicamentos.

\section{Etiopatogenia}

Los análisis filogenéticos han identificado 4 géneros de coronavirus diferentes $(\alpha, \beta, \gamma, \delta)$, siendo el SARS-CoV-2 un agente distinto y nuevo que forma parte del grupo de $\beta$-coronavirus ${ }^{3,4}$. Su estructura molecular es semejante a la de los coronavirus SARS-CoV (Severe Acute Respiratory Syndrome-CoronaVirus, manifestado en China el año 2002) y MERS-CoV (Middle East Respiratory Syndrome-Corona Virus, manifestado en el Medio Oriente el año 2012), con un genoma compuesto por 32 mil pares de bases ${ }^{4}$ y una transcriptasa inversa con una alta capacidad de realizar copias erróneas en el período infectivo. Esto repercute con elevadas tasas de recombinación de ARN, afectando particularmente a la proteína $\mathrm{S}$ (proteína de superficie), la que irá acumulando mutaciones, otorgándole una mayor antigenicidad dentro del huésped y un mayor grado de transmisibilidad $^{4}$. Actualmente, del total de mutaciones acumuladas, la mutación $\mathrm{D} 614 \mathrm{G}$ es la forma dominante de la proteína $\mathrm{S}$ que se ha expandido a nivel mundial ${ }^{5}$.

Las glicoproteínas que envuelven la superficie del SARS-CoV-2 son las que le dan el aspecto típico de "corona". De estas, la proteína $\mathrm{S}$ (del inglés Spike, espiga), que se compone de
2 subunidades denominadas S1 (subunidad de superficie) y S2 (subunidad transmembrana), es la que se une al receptor celular ECA-2 (enzima convertidora de angiotensina 2) a partir de un dominio de unión al receptor (RBD) de la proteína $\mathrm{S}$, asociada a una proteasa conocida como TMPRSS2. Esta proteasa producirá una escisión entre las subunidades S1 y S2, permitiendo que la subunidad S2 se ancle en la membrana celular de la célula huésped, endocitando el virus en la célula $^{6}$ y liberando el genoma de ARN viral monocatenario al citoplasma. Recientemente se está estudiando la participación de una glicoproteína transmembrana denominada NRP1 (neuropilina 1) como un tercer receptor para SARS-CoV-2; ya sea en su expresión única (donde desarrollaría detección viral a bajas concentraciones) o asociada a los receptores ECA-2 y TMPRSS2 (donde su infectividad se desarrollaría con más potencia) ${ }^{7}$. NRP1 se encontraría ubicada principalmente en células endoteliales del tejido nervioso ${ }^{7}$, lo que sería una posible explicación para entender los fenómenos neurológicos que se han estado reportando en relación al COVID-19.

El genoma del SARS-CoV-2, lo componen 5 regiones genómicas, de las cuales la región codificante de la enzima de replicación (compuesta por dos genes: ORF1a y ORF1b) y los genes estructurales $(\mathrm{S}, \mathrm{E}, \mathrm{M}$ y $\mathrm{N})$ tendrán una participación activa en la patogenia ${ }^{4}$. Los genes ORF1a y ORF1b expresan y codifican 2 poliproteínas que se proteolizan en 16 subproductos (incluyendo proteasas, helicasa, polimerasa y exonucleasa) ${ }^{8}$, dando origen a la transcriptasa inversa. La localización de esta enzima se ha hallado frecuentemente en las membranas intracelulares del retículo endoplásmico (RE), desde donde iniciará la replicación, transcripción y traducción viral; codificando en parte las proteínas estructurales S (espiga), M (membrana), E (envoltura) y N (nucleocápside). Estos subproductos virales se internalizarán en las membranas de los complejos Retículo Endoplásmico y Golgi, para ser exportados como glicoproteínas listas para su ensamblaje. Finalmente, será por medio de vesículas que estos virus se liberarán por exocitosis para continuar infectando el resto de las células pulmonares ${ }^{9}$.

A medida que se desarrolla la replicación y exocitosis viral, se han descrito fenómenos de piroptosis y apoptosis masiva de células epiteliales y endoteliales del pulmón, además del aumento de la permeabilidad vascular con infiltración de linfocitos; los que desencadenarán una masiva liberación de citocinas y quimiocinas proinflamatorias $^{10}$, dentro de las cuales la interleucina 
(IL)-6 (IL-6) será el principal mediador ${ }^{11}$. Este fenómeno estimulará a las células inmunes (células B, células T, natural killer, células dendríticas y monocitos) en un intento de neutralizar esta replicación viral activa desencadenada ${ }^{12}$, hasta un punto en el que se terminará potenciando la actividad inflamatoria y procoagulante, desarrollando lo que se conoce como síndrome de liberación de citoquinas (SLC); un fenómeno caracterizado por desarrollar una respuesta inflamatoria sistémica capaz de generar una amplia gama de manifestaciones clínicas secundarias a la disfunción de diversos sistemas orgánicos, alcanzando finalmente una falla multiorgánica (FMO $)^{11}$.

A nivel pulmonar, citoquinas como IL-1 $\beta$ (liberada durante la piroptosis), IL-6, IL-2, IL7 , IL-10, INF- $\gamma$ (interferón $\gamma$ ), TNF- $\alpha$ (factor de necrosis tumoral $\alpha$ ), G-CSF (factor estimulante de colonias de granulocitos), MCP1 (proteína quimiotáctica monocitaria 1) y MIP1 $\alpha$ (proteína inflamatoria de macrófagos $1 \alpha$ ) promoverán un aumento de la permeabilidad vascular, facilitando la salida de plasma y células sanguíneas a los alvéolos, instalando desde un daño pulmonar agudo hasta un síndrome de distrés respiratorio agudo (SDRA) ${ }^{11}$. Como consecuencia de este SLC proinflamatorio, se ha visto que a nivel de la microvasculatura pulmonar se desarrollan trombosis hialina difusa junto con fenómenos de ruptura endotelial con hemorragia secundaria, que potencian el SDRA que se está instalando ${ }^{13}$. Por otro lado, esta IL-6 al actuar como mediador central del SLC, tiene un rol importante en la disfunción multiorgánica que explica el compromiso cardiovascular (con disfunción e isquemia miocárdica), hepático, hematológico (con riesgo de desarrollar una coagulación intravascular diseminada, un síndrome antifosfolípidos o un tromboembolismo venoso), renal, neurológico, inmunológico y dermatológico que se han documentado hasta el momento ${ }^{11,13,14}$.

\section{Opciones farmacológicas de tratamiento}

La experiencia con los coronavirus SARSCoV y MERS-CoV, junto a estudios de actividad 'in vitro' y en modelos animales, han permitido sugerir agentes terapéuticos potenciales contra el SARS-CoV-2, cuya eficacia que no se ha logrado definir de forma clara debido a la escasa evidencia disponible y sus limitaciones metodológicas; sin embargo, la información en cuanto al manejo del COVID-19 está surgiendo continuamente ${ }^{15}$.

Se puede deducir que las dianas farmacológicas pueden aplicarse según el desarrollo de las fases de la infección: fase I (infección temprana), fase IIa (pulmonar sin hipoxia), fase IIb (pulmonar con hipoxia) y fase III (inflamatoria y procoagulante). Se cree que el uso de fármacos antivirales (Hidroxicloroquina, Lopinavir-Ritonavir, Umifenovir, Favipiravir, Remdesivir) en las etapas tempranas de la infección puede traer un posible beneficio versus su uso en fases tardías. Por otro lado, el uso de fármacos con actividad antiinflamatoria e inmunomoduladora (Corticoides, Tocilizumab, Anakinra e Inmunoglobulina Humana Intravenosa) se promueven con el fin el reducir los efectos del SLC previo a la FMO, no obstante, aún no existen estudios que respalden su seguridad ${ }^{16}$.

\section{Cloroquina e Hidroxicloroquina}

Ambas son bases débiles que podrían afectar la endocitosis y exocitosis viral al inhibir la acidificación endosómica y la glicosilación de los receptores del huésped, afectando además las etapas tardías de replicación viral al alterar la actividad proteolítica del genoma vira ${ }^{17}$. Los primeros estudios mostraron que la hidroxicloroquina tenía un efecto más potente que la cloroquina para inhibir el SARS-CoV-2 'in vitro" ${ }^{17}$, además de un posible un efecto anti-inflamatorio que reduciría la secreción de citoquinas pro-inflamatorias por parte de los macrófagos ${ }^{18}$, hecho que pudo beneficiar a los pacientes con SDRA.

Estudios posteriores evidenciaron que la hidroxicloroquina podría reducir la carga viral del SARS-CoV-2 en la nasofaringe de pacientes con COVID-19 a partir del tercer día de tratamiento, efecto que eventualmente se potenciaría al asociar azitromicina los siguientes 4 días; completando 5 días de terapia ${ }^{19}$. En similares condiciones, este mismo esquema mostraría resultados semejantes con dosis menores de hidroxicloroqui$\mathrm{na}^{20}$. No obstante, las limitaciones metodológicas de ambas investigaciones llevaron a que no se lograra adoptar estos esquemas como manejo estándar, sumado a la preocupación existente ante la toxicidad que pudiese asociar el uso de esta terapia combinada ${ }^{21}$. Incluso, un estudio de cohorte retrospectivo con 1.438 pacientes hospitalizados con COVID-19, donde se comparó el uso de hidroxicloroquina, azitromicina o ambos, versus ninguno de estos tratamientos; no mostró asociación significativa entre su uso y la mortalidad hospitalaria ${ }^{22}$. Otro estudio, con 96.032 pacientes con COVID-19, donde se compararon 14.888 pacientes que recibieron tratamiento (cloroquina sola, cloroquina con macrólido, hidroxicloroquina sola e hidroxicloroquina con macrólido) versus 81.144 pacientes como grupo control; no se 
pudo confirmar beneficio en disminuir la mortalidad hospitalaria con el uso de hidroxicloroquina o de cloroquina (tanto en su uso en solitario como asociada a macrólido), reportando afectación en la supervivencia hospitalaria de estos pacientes al desarrollar arritmias ventriculares secundarias al uso de estos fármacos ${ }^{23}$.

\section{Lopinavir-Ritonavir (Kaletra)}

Lopinavir es un inhibidor de la proteasa del VIH (virus de la inmunodeficiencia humana)-1, que tiene actividad inhibitoria 'in vitro' contra el SARS-CoV y MERS-CoV. Al asociarse a Ritonavir puede aumentar su vida media plasmática mediante la inhibición del citocromo $\mathrm{P} 450^{24}$. Si bien, no existen datos 'in vitro' de los efectos en SARS-CoV-2 ${ }^{21}$, un estudio comparó la actividad antiviral de estos fármacos frente a este agente ${ }^{25}$ y no asoció ningún beneficio tanto en casos graves $^{24}$ como en casos leves a moderados ${ }^{26}$, sin diferencias significativas en el aclaramiento viral o las tasas de mortalidad a los 28 días; no obstante, la mediana de inicio de esta terapia fue de 13 días. Si bien, en los grupos en que se inició tratamiento de forma precoz (antes de 12 días) se pudo advertir una mejoría clínica y una menor mortalidad, esta no alcanzó diferencias significativas. Otro estudio, con 127 pacientes hospitalizados por COVID-19 leve a moderado, comparó el tiempo de negativización de la rRT-PCR (reacción en cadena de la polimerasa en tiempo real con transcriptasa inversa) ante el uso combinado de Lopinavir/Ritonavir asociado a Ribavirina e Interferón $\beta 1 \mathrm{~b}$, versus el uso de Lopinavir/Ritonavir como grupo control. Esta negativización se logró en promedio durante 7 días (con un rango entre 5 a 11 días) con el uso de esta triterapia versus un promedio de 12 días en el grupo control (con un rango de 8 a 15 días) ${ }^{27}$.

El estudio RECOVERY (del acrónimo de Randomised Evaluation of COVid-19 thERapY), comparó una gama de opciones farmacológicas como terapia para el COVID-19, cuyos resultados preliminares pudieron concluir la ausencia de beneficio clínico del uso de hidroxicloroquina y de Lopinavir/Ritonavirir-30. Respecto a hidroxicloroquina, esta no logró reducir la mortalidad a 28 días (principal criterio a evaluar), pero sí se asoció a un mayor período de hospitalización y un mayor riesgo de progresar a ventilación mecánica invasiva (VMI) (ambos como criterios secundarios a evaluar) ${ }^{28}$. Por otro lado, en el grupo de Lopinavir/Ritonavir, 4\% de estos pacientes requirieron de VMI y 70\% requirió de oxigenoterapia, versus $26 \%$ que no precisó de asistencia ventilatoria. Si bien, no se observaron diferencias significativas en cuanto a mortalidad a los 28 días, esta terapia no evitó la necesidad de VMI, ni influyó en el tiempo de hospitalización, confirmando de este modo la ausencia de beneficio ${ }^{29}$.

\section{Remdesivir}

Ha mostrado eficacia en la inhibición de la replicación de una amplia gama de coronavirus, confirmando su actividad 'in vitro' en el SARS$\mathrm{CoV}-2^{30}$. Es un profármaco modificado análogo de nucleósido de adenina, que se incorpora a las cadenas de ARN virales nacientes y produce un término prematuro de su replicación. Sus usos podrían incluir tanto la profilaxis como el tratamiento para COVID-19 $9^{31}$. Estudios en MERS$\mathrm{CoV}$ han demostrado que la dosis de inhibición in vitro de Remdesivir es 100 veces más baja que la de Lopinavir-Ritonavir ${ }^{25}$. Un estudio multicéntrico, aleatorizado, doble ciego y controlado con placebo, con un total de 237 pacientes; de los cuales 158 pacientes fueron asignados a tratamiento con Remdesivir y 79 a placebo, mostró que el uso de Remdesivir no optimizó significativamente el tiempo de mejoría clínica, mortalidad o tiempo de eliminación del virus en pacientes con COVID-19 grave en comparación con placebo $^{32}$; sin embargo, este estudio no alcanzó el objetivo planteado debido a una disminución en el número de casos de pacientes con COVID-19, lo que redujo su impacto estadístico de $80 \%$ a $58 \%{ }^{32}$. Otro estudio aleatorizado, doble ciego, controlado con placebo y multicéntrico (con 68 centros inscritos: 47 en Estados Unidos y 21 en países de Europa y Asia) incluyó a 1.063 pacientes hospitalizados con COVID-19 severo y afectación pulmonar, con el objetivo de evaluar el tiempo de recuperación (definido por el alta hospitalaria o la hospitalización para control de infecciones). Los datos preliminares del análisis provisional (29.04.2020) mostraron que el grupo que recibió Remdesivir tuvo un tiempo de recuperación más rápido que los que recibieron placebo, pasando de una mediana de 15 días en el grupo control (con un rango de 13 a 19 días) a 11 días en el grupo que recibió el fármaco (con un rango de 9 a 12 días) ${ }^{33}$.

\section{Umifenovir (Arbidol) y Favipiravir}

Umifenovir es un agente antiviral con actividad contra el complejo que forma la proteína $\mathrm{S}$ y la ECA-2, impidiendo su internalización en la célula pulmonar. Es un fármaco prometedor para el tratamiento del COVID-19, según resultados 'in vitro' en estudios efectuados contra SARS-CoV, no obstante, son resultados en espera de publicar a partir de ensayos clínicos en curso $^{21}$. 
Por otro lado, Favipiravir es un profármaco, cuyo agente activo inhibe la ARN polimerasa y detiene por tanto la replicación viral. Su conocimiento proviene de la experiencia con Influenza y Ébola, mostrando actividad contra otros virus de $\mathrm{ARN}^{21}$.

Un ensayo prospectivo, aleatorizado, controlado, abierto y multicéntrico, comparó el uso de umifenovir versus favipiravir durante 10 días en 240 pacientes con COVID-19. Este mostró que el grupo con Favipiravir condujo a latencias más cortas en relación al alivio de la fiebre y la tos, pero sin mejoría significativa en la tasa de recuperación clínica ${ }^{34}$; en comparación al uso de umifenovir, dejando inconclusa la eficacia que estos fármacos pudiesen ofrecer.

\section{Ivermectina}

Es un agente antiparasitario de amplio espectro que ha demostrado actividad antiviral 'in vitro' en una gran variedad de virus (VIH-1, Dengue, $\mathrm{H} 1 \mathrm{~N} 1$, entre otros $)^{35}$. Actualmente, se está estudiando 'in vitro' la inhibición de la importación nuclear de las proteínas virales del SARS-CoV-2, con resultados que demuestran cierta acción antiviral con una dosis única capaz de controlar la replicación durante 24 a 48 h. Esto, sumado a la posibilidad de limitar la carga viral si se administra de forma temprana en las fases de infección ${ }^{35}$, lo hacen un medicamento prometedor en fase de estudio 'in vitro'. Pese a esto, las concentraciones antivirales para inhibir al SARS-CoV-2 sólo podrían alcanzarse a dosis superiores a la terapéutica (es decir, sobredosis masiva de este fármaco que no son posibles en humanos), haciendo poco recomendable su uso ${ }^{36}$.

\section{Interferones}

El interferón beta ( $\beta$-INF) es un agente antiviral e inmunomodulador que ha mostrado potencia contra el MERS-CoV ${ }^{37}$, observándose cierta actividad sinérgica con Ribavirina y Lopinavir/ Ritonavir $^{25,27}$ en el manejo del SARS-CoV-2. De momento, su uso ha sido estudiado sólo en asociación a otros fármacos, por lo que no se conoce realmente su grado de efectividad en el manejo del COVID-19.

\section{Corticoides}

Tienen un efecto inhibidor de la actividad fisiológica de las células $\mathrm{T}$ en relación al SLC, siendo utilizados con éxito en el manejo de este síndrome ${ }^{38}$, con beneficios en el manejo del SDRA no infeccioso. En la etapa IIa de la infección por SARS-CoV-2, es probable que su uso no tenga beneficio alguno; sin embargo, se cree que pueden tener un rol activo en la etapa $\mathrm{IIb}$, dado que la hipoxia hace necesario el uso de ventilación mecánica, ante el cual la terapia corticoidal podría emplearse con prudencia por períodos cortos para reducir la duración de la ventilación y la mortalidad general ${ }^{16}$. La experiencia obtenida del SARS-CoV y MERS-CoV concluyó que el uso inicial de pulsos con dosis elevadas de corticoides puede ser beneficioso, pese a que estos fármacos aumentan la viremia y retrasan el clearence viral ${ }^{39}$. Otros investigadores han concluido que existe mayor probabilidad de beneficio tras su uso en infecciones bacterianas más que por infecciones virales ${ }^{40}$; como en la neumonía por influenza en la que se ha detectado un aumento de la mortalidad tras su empleo ${ }^{39}$. En contraste a esto, un estudio observacional retrospectivo, con 55 pacientes con COVID-19, donde estratificaron estos pacientes según gravedad (leve, moderado y severo) para aplicar distintos esquemas terapéuticos, mostró que en aquellos pacientes con COVID-19 grave, el uso temprano de corticoides (idealmente al ingreso o las primeras $72 \mathrm{~h}$ de hospitalización), a dosis bajas y a corto plazo no retrasó el clearance viral como se tenía descrito, influyendo además en la producción de anticuerpos de tipo IgG al compararlos con los pacientes no graves ${ }^{41}$. Esta discrepancia de resultados ha llevado a no establecer indicaciones definitivas sobre el uso de corticoides en pacientes con COVID-19. Así, su recomendación se mantiene a la espera de que los ensayos en marcha puedan responder sobre su utilidad en la respuesta inflamatoria instalada tras la primera fase de la infección ${ }^{11}$.

En este sentido, tras la publicación de los resultados preliminares del estudio RECOVERY, la determinación uso de corticoides en COVID-19 ha podido definir un beneficio concreto en cuanto a reducir el riesgo de mortalidad ${ }^{42}$, basándose en la hipótesis de que los glucocorticoides pueden modular la lesión pulmonar mediada por SLC, permitiendo, por lo tanto, reducir la progresión a insuficiencia respiratoria y muerte. Para ello, se incluyó una muestra de 6.425 pacientes hospitalizados (tanto con sospecha para COVID-19 o con diagnóstico confirmado por laboratorio), utilizando como terapia $6 \mathrm{mg}$ de dexametasona al día (de uso oral o intravenoso) por un período máximo de 10 días de tratamiento; con el objetivo de valorar la mortalidad a 28 días (como criterio principal), el tiempo requerido hasta el alta hospitalaria y la necesidad de uso de VMI (ambos como criterios secundarios). De los 6.425 pacientes analizados, 2.104 fueron incluidos en el grupo de tratamiento y 4.321 en el grupo control, de 
los cuales, un total de 482 pacientes $(22,9 \%)$ del grupo de tratamiento y 1.110 pacientes $(25,7 \%)$ del grupo control murieron a los 28 días de iniciada la aleatorización (con un cociente de tasas ajustado por edad, 0,83; intervalo de confianza [IC] del 95\%, 0,75 a 0,93; $\mathrm{p}<0,001)$. En el grupo de tratamiento, la incidencia de muerte fue menor que en el grupo control; entre los pacientes que recibieron VMI $(29,3 \%$ frente al $41,4 \%$; riesgo relativo, 0,64; IC $95 \%, 0,51-0,81$ ) y entre los que recibieron oxígeno sin VMI (23,3 versus $26,2 \%$; riesgo relativo, 0,82; IC $95 \%, 0,72-0,94)$. No fue posible demostrar una relación similar en aquellos que no recibían asistencia respiratoria al momento de la aleatorización $(17,8 \%$ frente a $14,0 \%$; riesgo relativo, 1,19 ; IC $95 \%, 0,91-1,55)$. Por lo tanto, con estos resultados preliminares, es posible concluir que el uso de dexametasona se asocia a una menor mortalidad a los 28 días, logrando además una mediana de tiempo de hospitalización menor (12 versus 13 días), una probabilidad mayor de lograr el alta hospitalaria en un período de 28 días (riesgo relativo, 1,10; IC $95 \%, 1,03-1,17$ ) y una frecuencia menor de uso de VMI desde la aleatorización (riesgo relativo, 0,77; IC $95 \%, 0,62-0,95)$, destacando además que, en pacientes que no requieren de oxigenoterapia, no debiese emplearse el uso de dexametasona dado que existe una tendencia a aumentar la mortalidad $^{42}$.

\section{Antagonistas de la IL-6 (Tocilizumab y Sarilumab) e IL-1 (Anakinra)}

Tocilizumab es un anticuerpo monoclonal conocido por su efecto inhibidor del receptor de interleucina 6 (IL-6), que podría tener efectividad en el SLC secundario a COVID-19 grave $^{11}$. Si bien, es un fármaco que permanece en investigación, hay estudios que reportan mejoría clínica en COVID-19 grave $\left(\mathrm{SaO}_{2} \quad 93 \%\right.$ o $\mathrm{PaO}_{2} / \mathrm{FiO}_{2}$ $<300 \mathrm{mmHg}$ ) que poseen niveles elevados de IL-6 ${ }^{11}$. Entre estos, un estudio observacional de 21 pacientes mostró mejoría clínica en el 91\% de ellos; la mayoría con una sola dosis de Tocilizumab $^{43}$. Sin embargo, la ausencia de un grupo comparativo ha limitado la interpretación de estos resultados. Un estudio piloto abierto multicéntrico, con una sola línea de estudio sobre el uso de Tocilizumab, con 63 pacientes hospitalizados por COVID-19 severo, observó una mejoría significativa en los niveles de ferritina, proteína $\mathrm{C}$ reactiva, dímero $\mathrm{D}$ y $\mathrm{PaO}_{2} / \mathrm{FiO}_{2}$, aumentando la supervivencia hospitalaria de estos pacientes a $89 \%$ y alcanzando un mejor pronóstico en aquellos donde su administración ocurrió los primeros 6 días tras el ingreso ${ }^{44}$. Pese a esto, hacen falta ensayos controlados para confirmar un beneficio definitivo de este fármaco.

Sarilumab es otro antagonista de la IL-6 que está siendo estudiado bajo un ensayo multicéntrico, doble ciego, en fase 2 para pacientes con COVID-19 grave $^{45}$; que compara el uso de Sarilumab (bajo dosis de $200 \mathrm{mg}$ y $400 \mathrm{mg}$ ) versus placebo. Resultados preliminares no han mostrado diferencias a dosis de $200 \mathrm{mg}$ versus placebo, por lo que la continuidad del estudio en la fase 3 será sólo con dosis de $400 \mathrm{mg}$ en pacientes graves ${ }^{45}$.

Anakinra es un antagonista recombinante del receptor de IL-1, que bloquea la actividad de las citocinas proinflamatorias IL-1 $\alpha$ e IL- $1 \beta^{46}$ expresadas en el SLC. Un estudio de cohorte retrospectivo comparó el uso de anakinra en 36 pacientes, a dosis altas ( $5 \mathrm{mg}$ por vía intravenosa, dos veces al día) y dosis bajas (100 mg por vía subcutánea, dos veces al día), versus un grupo control (siendo suspendida la rama de dosis bajas en los 7 pacientes que la utilizaron por ausencia de eficacia ${ }^{46}$. A los 21 días, el tratamiento con dosis altas de anakinra se asoció con reducciones de parámetros de laboratorio y mejora progresiva en la función respiratoria en $21(72 \%)$ de los 29 pacientes restantes que estaban utilizando este fármaco, alcanzando una supervivencia del $90 \%$ en el grupo de dosis altas de anakinra versus un $56 \%$ en el grupo control ${ }^{46}$. Nuevamente, se requieren ensayos controlados para confirmar un beneficio definitivo de este fármaco.

\section{Plasma de convaleciente}

Es un tipo de inmunoterapia basada en anticuerpos específicos del SARS-CoV-2, presentes en el plasma convaleciente de pacientes recuperados. Su mecanismo de acción se cree que tiene relación con la neutralización directa del virus (mediante anticuerpos neutralizantes), el control de un sistema inmunitario hiperactivo (mediante linfocitos Th1/Th17 y la activación de la cascada del complemento) y la inmunomodulación de los estados hipercoagulables ${ }^{47}$. Este recurso se utilizó como última medida contra enfermedades infecciosas severas (SARS-CoV, MERS-CoV, Virus Ébola, Influenza A, H1N1), demostrando reducción de la estancia hospitalaria y mortalidad si se administra principalmente dentro de los primeros 7 a 10 días de la infección ${ }^{48}$. A pesar de esto, en COVID-19 aún no se tiene claridad respecto si proporciona o no algún beneficio clínico ${ }^{48}$. Una serie de casos no controlados de 5 pacientes críticos con COVID-19 con SDRA en VMI, mostró como hallazgos preliminares una mejora en el estadio clínico tras la administración de plasma convaleciente $^{49}$, agregando una disminución de 
la carga viral y la resolución del SDRA (en 4 de los 5 pacientes) a los 12 días de administrada la transfusión de plasma ${ }^{49}$. Si bien, es imposible generar una conclusión sobre la efectividad de esta terapia, estas observaciones motivan la evaluación mediante ensayos clínicos.

\section{Terapia Anticoagulante}

Parte de la patogenia son los fenómenos protrombóticos que se desarrollan a causa del SLC generado por el SARS-CoV-2, cuyo impacto repercute en el compromiso cardiopulmonar de estos pacientes ${ }^{13}$. Un estudio, con 449 pacientes con COVID-19 grave comparó la mortalidad a los 28 días entre los usuarios de heparina (99 pacientes) y los no usuarios (350 pacientes), versus el riesgo de coagulopatía; estratificada por puntaje de coagulopatía inducida por sepsis (SIC) o el resultado del dímero D. Si bien, no se encontraron diferencias en cuanto a mortalidad a 28 días (30,3 versus $29,7 \%, \mathrm{p}=0,910)$, sí se encontró una mortalidad más baja en pacientes con puntaje $\mathrm{SIC} \geq 4(40,0$ versus $64,2 \%, \mathrm{p}=0,029)$, pero no en aquellos con puntaje SIC $<4(29,0$ versus $22,6 \%, p=0,419)$. Al analizar los valores de dímero $\mathrm{D}$, con valores mayores a $3,0 \mu \mathrm{g} / \mathrm{mL}$ (6 veces el límite superior normal), sí se encontró una reducción aproximada del $20 \%$ en la mortalidad con tratamiento con heparina $(32,8 \%$ versus $52,4 \%, p=0,017)$. Si bien, estos resultados no mostraron significancia estadística, pareciera que el uso de heparina ofrece un mejor pronóstico en pacientes con COVID-19 grave, que cumplen con los criterios de SIC o con dímero D marcadamente elevado ${ }^{50}$. Un segundo estudio, de tipo observacional, con 2.773 pacientes hospitalizados por COVID-19, evaluó el efecto de la dosis sistémica de tratamiento anticoagulante (incluida la forma oral, subcutánea o intravenosa) sobre la mortalidad hospitalaria en 786 de estos pacientes (que tenían una probabilidad mayor de requerir VMI en el contexto de un escenario clínico más grave $)^{51}$, observando que la mayor duración del tratamiento anticoagulante, en pacientes hospitalizados en UCI y en ventilación mecánica, se asoció con una mejora en la supervivencia, con un disminución del riesgo de mortalidad ${ }^{51}$.

\section{Otras terapias}

La diana inmunológica se está abriendo campo a nuevas posibilidades terapéuticas; es así como estudios preliminares han nombrado al anticuerpo monoclonal humano contra un epítopo común que bloquea la infección por SARS-COV-2 como una de las innovaciones en desarrollo ${ }^{20}$. Otros fármacos en estudio son los inhibidores del
Complejo JAK (Baricitinib) ${ }^{52}$ y la Colchicina ${ }^{53}$. Esta última, ejerce un efecto antiinflamatorio directo al inhibir la síntesis del TNF- $\alpha$ e IL-6, la migración de monocitos y la secreción de metaloproteinasas; por lo que se cree pueda haber efecto en el SLC.

\section{Conclusiones}

El conocimiento de la patogenia del SARSCoV-2 y de las fases clínicas del COVID-19, han permitido caracterizar de mejor manera el modo de dirigir el tratamiento. No obstante, muchos de los resultados de ensayos clínicos han sido poco favorecedores al momento de definir un esquema terapéutico específico. La evidencia disponible ha sido dinámica, y en cierto grado conflictiva, al momento de referirse a una terapia antiviral (en especial entre hidroxicloroquina, lopinavir/ ritonavir y remdesivir,) o el uso de fármacos antiinflamatorios e inmunomoduladores. Sin embargo, no se descarta que cambien nuevamente estas recomendaciones, pues existen ensayos clínicos en curso, cuyas conclusiones logren modificar el actual paradigma.

\section{Bibliografía}

1.- WHO Director-General's opening remarks at the media briefing on COVID-19 - 11 March 2020 [Internet]. [citado el 6 de mayo de 2020]. Disponible en: https:// www.who.int/dg/speeches/detail/who-director-generals-opening-remarks-at-the-media-briefing-on-covid19---11-march-2020.

2.- ZHU N, ZHANG D, WANG W, LI X, YANG B, SONG J, et al. A Novel Coronavirus from Patients with Pneumonia in China, 2019. N Engl J Med [Internet]. 20 de febrero de 2020 [citado el 6 de mayo de 2020]; 382 (8): 727-33. Disponible en: http://www.nejm.org/ doi/10.1056/NEJMoa2001017.

3.- SHEREEN MA, KHAN S, KAZMI A, BASHIR N, SIDDIQUE R. COVID-19 infection: Origin, transmission, and characteristics of human coronaviruses. J Adv Res [Internet]. 1 de julio de 2020 [citado el 6 de mayo de 2020]; 24: 91-8. Disponible en: https://www.sciencedirect.com/science/article/pii/S2090123220300540.

4.- LI X, GENG M, PENG Y, MENG L, LU S. Molecular immune pathogenesis and diagnosis of COVID-19. J Pharm Anal [Internet]. 1 de abril de 2020 [citado el 6 de mayo de 2020]; 10 (2): 102-8. Disponible en: https://linkinghub.elsevier.com/retrieve/pii/S2095177920302045.

5.- KORBER B, FISCHER W, GNANAKARAN SG, YOON H, THEILER J, ABFALTERER W, et al. Spike mutation pipeline reveals the emergence of a 
more transmissible form of SARS-CoV-2. bioRxiv [Internet]. 2020; 2020.04.29.069054. Disponible en: https://www.biorxiv.org/content/10.1101/2020.04.29.0 69054v2.

6.- MENG T, CAO H, ZHANG H, KANG Z, XU D, GONG $\mathrm{H}$, et al. The insert sequence in SARS-CoV-2 enhances spike protein cleavage by TMPRSS. bioRxiv [Internet]. 2020;2020.02.08.926006. Disponible en: https://www.biorxiv.org/content/10.1101/2020.02.08.9 26006v3.

7.- CANTUti-CASTELVETRI L, OHJA R, PEDRO L, DJANNATIAN M, FRANZ J, KUIVANEN S, et al. Neuropilin-1 facilitates SARS-CoV-2 cell entry and provides a possible pathway into the central nervous system. bioRxiv [Internet]. 1 de enero de 2020; 2020.06.07.137802. Disponible en: http://biorxiv.org/ content/early/2020/06/10/2020.06.07.137802.abstract.

8.- WEISS SR, LEIBOWITZ JL. Coronavirus Pathogenesis. En: Advances in Virus Research [Internet]. Academic Press Inc. 2011 [citado el 6 de mayo de 2020]; 85-164. Disponible en: https://www.ncbi.nlm.nih.gov/ pmc/articles/PMC7149603/.

9.- DE WIT E, VAN DOREMALEN N, FALZARANO D, MUNSTER VJ. SARS and MERS: recent insights into emerging coronaviruses. Nat Rev Microbiol [Internet]. 27 de agosto de 2016 [citado el 6 de mayo de 2020]; 14 (8): 523-34. Disponible en: http://www. nature.com/articles/nrmicro.2016.81.

10.- YAN R, ZHANG Y, LI Y, XIA L, GUO Y, ZHOU Q. Structural basis for the recognition of SARS-CoV-2 by full-length human ACE2. Science (80-) [Internet]. 27 de marzo de 2020 [citado el 12 de mayo de 2020]; 367 (6485): 1444-8. Disponible en: https://www.sciencemag. org/lookup/doi/10.1126/science.abb2762

11.- ZHANG C, WU Z, LI J-W, ZHAO H, WANG G-Q. Cytokine release syndrome in severe COVID-19: interleukin- 6 receptor antagonist tocilizumab may be the key to reduce mortality. Int $\mathrm{J}$ Antimicrob Agents [Internet]. 29 de mayo de 2020 [citado el 6 de mayo de 2020]; 55 (5): 105954. Disponible en: https://linkinghub.elsevier. com/retrieve/pii/S0924857920301047.

12.- WU F, WANG A, LIU M, WANG Q, CHEN J, XIA S, et al. Neutralizing antibody responses to SARS-CoV-2 in a COVID-19 recovered patient cohort and their implications. medRxiv [Internet]. 1 de enero de 2020; 2020.03.30.20047365. Disponible en: http://medrxiv. org/content/early/2020/04/20/2020.03.30.20047365. abstract.

13.- MCGONAGLE D, O'DONNELL J, SHARIF K, EMERY P, BRIDGEWOOD C. Immune Mechanisms of Pulmonary Intravascular Coagulopathy (PIC) in COVID-19 Pneumonia. Lancet Rheumatol [Internet]. 2020; 2019 (20):1-9. Disponible en: https://www.researchgate. net/publication/340621484_Why_the_Immune_Mechanisms_of_Pulmonary_Intravascular_Coagulopathy_in COVID-19_Pneumonia_are_Distinct_from_Macropha-
ge_Activation_Syndrome_with_Disseminated_Intravascular_Coagulation.

14.- GALVÁN CASAS C, CATALÀ A, CARRETERO HERNÁNDEZ G, RODRÍGUEZ-JIMÉNEZ P, FERNÁNDEZ NIETO D, RODRÍGUEZ-VILLA LARIO A, et al. Classification of the cutaneous manifestations of COVID-19: a rapid prospective nationwide consensus study in Spain with 375 cases. Br J Dermatol [Internet]. 29 de abril de 2020; bjd.19163. Disponible en: https://onlinelibrary.wiley.com/doi/abs/10.1111/ bjd.19163.

15.- THORLUND K, DRON L, PARK J, HSU G, FORREST JI, MILLS EJ. A real-time dashboard of clinical trials for COVID-19. Lancet Digit Heal [Internet]. junio de 2020 [citado el 12 de mayo de 2020]; 2 (6): e286-7. Disponible en: https://linkinghub.elsevier.com/ retrieve/pii/S2589750020300868.

16.- SIDDIQI HK, MEHRA MR. COVID-19 illness in native and immunosuppressed states: A clinical-therapeutic staging proposal. J Hear Lung Transplant [Internet]. 1 de mayo de 2020 [citado el 6 de mayo de 2020]; 39 (5): 405-7. Disponible en: https://linkinghub.elsevier.com/ retrieve/pii/S105324982031473X.

17.- In Vitro Antiviral Activity and Projection of Optimized Dosing Design of Hydroxychloroquine for the Treatment of Severe Acute Respiratory Syndrome Coronavirus 2 (SARS-CoV-2) | Clinical Infectious Diseases | Oxford Academic [Internet]. [citado el 12 de mayo de 2020]. Disponible en: https://academic.oup.com/cid/ advance-article/doi/10.1093/cid/ciaa237/5801998.

18.- SAVARINO A, BOELAERT JR, CASSONE A, MAJORI G, CAUDA R. Effects of chloroquine on viral infections: an old drug against today's diseases. Lancet Infect Dis [Internet]. 1 de noviembre de 2003 [citado el 12 de mayo de 2020]; 3 (11): 722-7. Disponible en: https://linkinghub.elsevier.com/retrieve/pii/ S1473309903008065.

19.- GAUTRET P, LAGIER J-C, PAROLA P, HOANG VT, MEDDEB L, MAILHE M, et al. Hydroxychloroquine and azithromycin as a treatment of COVID-19: results of an open-label non-randomized clinical trial. Int $\mathrm{J}$ Antimicrob Agents [Internet]. 20 de marzo de 2020 [citado el 12 de mayo de 2020]; 105949. Disponible en: https://linkinghub.elsevier.com/retrieve/pii/ S0924857920300996.

20.- GAUTRET P, LAGIER J-C, PAROLA P, HOANG VT, MEDDEB L, SEVESTRE J, et al. Clinical and microbiological effect of a combination of hydroxychloroquine and azithromycin in 80 COVID-19 patients with at least a six-day follow up: A pilot observational study. Travel Med Infect Dis [Internet]. 1 de marzo de 2020 [citado el 12 de mayo de 2020]; 34: 101663. Disponible en: https://linkinghub.elsevier.com/retrieve/pii/ S1477893920301319.

21.- SANDERS JM, MONOGUE ML, JODLOWSKI TZ, CUTRELL JB. Pharmacologic Treatments for Corona- 
virus Disease 2019 (COVID-19). JAMA [Internet]. 13 de abril de 2020 [citado el 12 de mayo de 2020]; 323 (18): 1824-36. Disponible en: https://jamanetwork.com/ journals/jama/fullarticle/2764727.

22.- ROSENBERG ES, DUFORT EM, UDO T, WILBERSCHIED LA, KUMAR J, TESORIERO J, et al. Association of Treatment With Hydroxychloroquine or Azithromycin With In-Hospital Mortality in Patients With COVID-19 in New York State. JAMA [Internet]. 11 de mayo de 2020 [citado el 12 de mayo de 2020]; Disponible en: http://www.ncbi.nlm.nih.gov/pub$\mathrm{med} / 32392282$

23.- MEHRA MR, DESAI SS, RUSCHITZKA F, PATEL AN. Hydroxychloroquine or chloroquine with or without a macrolide for treatment of COVID-19: a multinational registry analysis. Lancet [Internet]. mayo de 2020; 6736 (20): 1-10. Disponible en: http://www. ncbi.nlm.nih.gov/pubmed/32450107.

24.- CAO B, WANG Y, WEN D, LIU W, WANG J, FAN $\mathrm{G}$, et al. A Trial of Lopinavir-Ritonavir in Adults Hospitalized with Severe Covid-19. N Engl J Med [Internet]. 7 de mayo de 2020 [citado el 12 de mayo de 2020]; 382 (19): 1787-99. Disponible en: http://www.nejm.org/ doi/10.1056/NEJMoa2001282.

25.- SHEAHAN TP, SIMS AC, LEIST SR, SCHÄFER A, WON J, BROWN AJ, et al. Comparative therapeutic efficacy of remdesivir and combination lopinavir, ritonavir, and interferon beta against MERS-CoV. Nat Commun [Internet]. 10 de diciembre de 2020 [citado el 12 de mayo de 2020]; 11 (1): 222. Disponible en: http:// www.nature.com/articles/s41467-019-13940-6.

26.- LI Y, XIE Z, LIN W, CAI W, WEN C, GUAN Y, et al. An exploratory randomized, controlled study on the efficacy and safety of lopinavir/ritonavir or arbidol treating adult patients hospitalized with mild/ moderate COVID-19 (ELACOI). medRxiv [Internet]. 15 de abril de 2020 [citado el 12 de mayo de 2020];2020.03.19.20038984. Disponible en: https:// www.medrxiv.org/content/10.1101/2020.03.19.200389 $84 \mathrm{v} 1$.

27.- HUNG IF-N, LUNG K-C, TSO EY-K, LIU R, CHUNG TW-H, CHU M-Y, et al. Triple combination of interferon beta-1b, lopinavir-ritonavir, and ribavirin in the treatment of patients admitted to hospital with COVID-19: an open-label, randomised, phase 2 trial. Lancet [Internet]. mayo de 2020; 6736 (20): 1-10. Disponible en: http://dx.doi.org/10.1016/S01406736(20)31042-4.

28.- HORBY P, MAFHAM M, LINSELL L, BELL JL, STAPLIN N, EMBERSON JR, et al. Effect of Hydroxychloroquine in Hospitalized Patients with COVID-19: Preliminary results from a multi-centre, randomized, controlled trial. medRxiv [Internet]. 15 de julio de 2020 [citado el 12 de agosto de 2020];2020.07.15.20151852. Disponible en: https://doi.org/10.1101/2020.07.15.2015 1852.
29.- Lopinavir-Ritonavir results-RECOVERY Trial [Internet]. [citado el 12 de agosto de 2020]. Disponible en: https://www.recoverytrial.net/results/lopinavar-results.

30.- WANG M, CAO R, ZHANG L, YANG X, LIU J, XU $\mathrm{M}$, et al. Remdesivir and chloroquine effectively inhibit the recently emerged novel coronavirus (2019-nCoV) in vitro [Internet]. Vol. 30, Cell Research. Springer Nature. 2020 [citado el 12 de mayo de 2020]; 269-71. Disponible en: https://www.nature.com/articles/s41422020-0282-0.

31.- DE WIT E, FELDMANN F, CRONIN J, JORDAN R, OKUMURA A, THOMAS T, et al. Prophylactic and therapeutic remdesivir (GS-5734) treatment in the rhesus macaque model of MERS-CoV infection. Proc Natl Acad Sci U S A [Internet]. 24 de marzo de 2020 [citado el 12 de mayo de 2020]; 117 (12): 6771-6. Disponible en: https://www.pnas.org/content/117/12/6771.long.

32.- WANG Y, ZHANG D, DU G, DU R, ZHAO J, JIN $\mathrm{Y}$, et al. Remdesivir in adults with severe COVID-19: a randomised, double-blind, placebo-controlled, multicentre trial. Lancet [Internet]. mayo de 2020; 395 (10236): 1569-78. Disponible en: http://dx.doi.org/10.1016/ S0140-6736(20)31022-9.

33.- BEIGEL JH, TOMASHEK KM, DODD LE, MEHTA AK, ZINGMAN BS, KALIL AC, et al. Remdesivir for the Treatment of COVID-19 - Preliminary Report. N Engl J Med [Internet]. 22 de mayo de 2020; NEJMoa2007764. Disponible en: https://doi.org/10.1056/ NEJMoa2007764

34.- CHEN C, ZHANG Y, HUANG J, YIN P, CHENG $\mathrm{Z}$, WU J, et al. Favipiravir versus Arbidol for COVID-19: A Randomized Clinical Trial. medRxiv [Internet]. 1 de enero de 2020;2020.03.17.20037432. Disponible en: http://medrxiv.org/content/ear ly/2020/04/15/2020.03.17.20037432.abstract.

35.- CALY L, DRUCE JD, CATTON MG, JANS DA, WAGSTAFF KM. The FDA-approved drug ivermectin inhibits the replication of SARS-CoV-2 in vitro. Antiviral Res [Internet]. 3 de abril de 2020 [citado el 12 de mayo de 2020]; 178: 104787. Disponible en: http:// www.ncbi.nlm.nih.gov/pubmed/32251768.

36.- MOMEKOV G, MOMEKOVA D. Ivermectin as a potential COVID-19 treatment from the pharmacokinetic point of view: antiviral levels are not likely attainable with known dosing regimens. medRxiv [Internet]. 1 de enero de 2020; 2020.04.11.20061804. Disponible en: http://medrxiv.org/content/ear ly/2020/05/22/2020.04.11.20061804.abstract.

37.- Lack of evidence to support use of darunavir-based treatments for SARS-CoV-2 | Janssen Ireland [Internet]. [citado el 12 de mayo de 2020]. Disponible en: https:// www.janssen.com/ireland/lack-evidence-support-usedarunavir-based-treatments-sars-cov-2 .

38.- RIEGLER LL, JONES GP, LEE DW. Current approaches in the grading and management of cytokine release syndrome after chimeric antigen receptor T-cell therapy. 
Ther Clin Risk Manag [Internet]. febrero de 2019 [citado el 12 de mayo de 2020]; Vol 15: 323-35. Disponible en: https://www.dovepress.com/current-approaches-inthe-grading-and-management-of-cytokine-release-speer-reviewed-article-TCRM.

39.- VILLAR J, FERRANDO C, MARTÍNEZ D, AMBRÓS A, MUÑOZ T, SOLER JA, et al. Dexamethasone treatment for the acute respiratory distress syndrome: a multicentre, randomised controlled trial. Lancet Respir Med [Internet]. 1 de marzo de 2020 [citado el 12 de mayo de 2020]; 8 (3): 267-76. Disponible en: https://linkinghub.elsevier.com/retrieve/pii/S2213260019304175.

40.- RUSSELL CD, MILLAR JE, BAILLIE JK. Clinical evidence does not support corticosteroid treatment for 2019-nCoV lung injury. Lancet (London, England) [Internet]. 15 de febrero de 2020 [citado el 12 de mayo de 2020]; 395 (10223): 473-5. Disponible en: http://www. ncbi.nlm.nih.gov/pubmed/32043983.

41.- ZHENG C, WANG J, GUO H, LU Z, MA Y, ZHU Y, et al. Risk-adapted Treatment Strategy For COVID-19 Patients. Int J Infect Dis [Internet]. mayo de 2020; 94: 74-7. Disponible en: https://doi.org/10.1016/j. ijid.2020.03.047.

42.- RECOVERY Collaborative Group, Horby P, Lim WS, Emberson JR, Mafham M, Bell JL, et al. Dexamethasone in Hospitalized Patients with Covid-19 - Preliminary Report. N Engl J Med [Internet]. 17 de julio de 2020; NEJMoa2021436. Disponible en: http://www.ncbi.nlm. nih.gov/pubmed/32678530

43.- XU X, HAN M, LI T, SUN W, WANG D, FU B, et al. Effective treatment of severe COVID-19 patients with tocilizumab. Proc Natl Acad Sci [Internet]. 19 de mayo de 2020; 117 (20): 10970-5. Disponible en: http://www. pnas.org/lookup/doi/10.1073/pnas.2005615117

44.- SCIASCIA S, APRÀ F, BAFFA A, BALDOVINO S, BOARO D, BOERO R, et al. Pilot prospective open, single-arm multicentre study on off-label use of tocilizumab in patients with severe COVID-19. Clin Exp Rheumatol [Internet]. 2020; 38 (3): 529-32. Disponible en: http://www.ncbi.nlm.nih.gov/pubmed/32359035.

45.- NCT04315298. Evaluation of the Efficacy and Safety of Sarilumab in Hospitalized Patients With COVID-19. https://clinicaltrials.gov/show/NCT04315298 [Internet]. 2020; Disponible en: https://www.clinicaltrials.gov/ct2/ show/NCT04315298.

46.- CAVAlli G, DE LUCA G, CAMPOCHIARO C, DELLA-TORRE E, RIPA M, CANETTI D, et al.
Interleukin-1 blockade with high-dose anakinra in patients with COVID-19, acute respiratory distress syndrome, and hyperinflammation: a retrospective cohort study. Lancet Rheumatol [Internet]. junio de 2020; 2 (6): e325-31. Disponible en: http://dx.doi.org/10.1016/ S2665-9913(20)30127-2.

47.- ROJAS M, RODRÍGUEZ Y, MONSALVE DM, ACOSTA-AMPUDIA Y, CAMACHO B, GALLO JE, et al. Convalescent plasma in COVID-19: Possible mechanisms of action. Autoimmun Rev [Internet]. mayo de 2020; (April): 102554. Disponible en: https://doi. org/10.1016/j.autrev.2020.102554.

48.- CHEN L, XIONG J, BAO L, SHI Y. Convalescent plasma as a potential therapy for COVID-19. Lancet Infect Dis [Internet]. 1 de abril de 2020 [citado el 12 de mayo de 2020]; 20 (4): 398-400. Disponible en: https://linkinghub.elsevier.com/retrieve/pii/ S1473309920301419.

49.- SHEN C, WANG Z, ZHAO F, YANG Y, LI J, YUAN J, et al. Treatment of 5 Critically Ill Patients With COVID-19 With Convalescent Plasma. JAMA [Internet]. 28 de abril de 2020; 323 (16): 1582. Disponible en: https://doi.org/10.1001/jama.2020.4783.

50.- TANG N, BAI H, CHEN X, GONG J, LI D, SUN Z. Anticoagulant treatment is associated with decreased mortality in severe coronavirus disease 2019 patients with coagulopathy. J Thromb Haemost [Internet]. 27 de mayo de 2020 [citado el 12 de mayo de 2020]; 18 (5): 1094-9. Disponible en: https://onlinelibrary.wiley.com/ doi/abs/10.1111/jth.14817.

51.- PARANJPE I, FUSTER V, LALA A, RUSSAK A, GLICKSBERG BS, LEVIN MA, et al. Association of Treatment Dose Anticoagulation with In-Hospital Survival Among Hospitalized Patients with COVID-19. J Am Coll Cardiol [Internet]. 6 de mayo de 2020; 27327. Disponible en: http://www.onlinejacc.org/content/ early/2020/05/05/j.jacc.2020.05.001.abstract.

52.- Treatment of Moderate to Severe Coronavirus Disease (COVID-19) in Hospitalized Patients - Full Text View - ClinicalTrials.gov [Internet]. [citado el 12 de mayo de 2020]. Disponible en: https://clinicaltrials.gov/ct2/show/ NCT04321993.

53.- The ECLA PHRI COLCOVID Trial. Effects of Colchicine on Moderate/High-risk Hospitalized COVID-19 Patients. - Full Text View - ClinicalTrials.gov [Internet]. [citado el 12 de mayo de 2020]. Disponible en: https:// clinicaltrials.gov/ct2/show/NCT04328480.
Correspondencia a:

Dr. Bastián Abarca Rozas

Lota 2465, Providencia, Chile. Código Postal 7500000

ORCID: https://orcid.org/0000-0002-7643-9496;

Email: fds.baar@gmail.com 